Gynecologic and

Obstetric Investigation
Gynecol Obstet Invest 2019;84:326-333

DOI: $10.1159 / 000495614$
Received: August 14, 2018

Accepted after revision: November 19, 2018

Published online: January 2, 2019

\title{
Second Trimester Serum Biomarker Screen for Fetal Aneuploidies as a Predictor of Preterm Delivery: A Population-Based Study
}

\author{
Sujinun Nunthapiwat Ratanaporn Sekararithi Chanane Wanapirak \\ Supatra Sirichotiyakul Fuanglada Tongprasert Kasemsri Srisupundit \\ Suchaya Luewan Theera Tongsong \\ Department of Obstetrics and Gynecology, Faculty of Medicine, Chiang Mai University, Chiang Mai, Thailand
}

\section{Keywords}

Alpha-fetoprotein - Beta-human chorionic gonadotropin · Early preterm delivery · Preterm delivery · Second trimester screening · Unconjugated estriol

\footnotetext{
Abstract

Objective: To determine the association between secondtrimester serum Down syndrome screening (alpha-fetoprotein [AFP] free beta-human chorionic gonadotropin [b-hCG] unconjugated estriol [uE3]) and preterm birth and to create predictive models for preterm birth. Methods: Secondary analysis on a prospective database of pregnancies undergoing second-trimester screen with complete follow-up. The multiples of medians (MoM) of the biomarkers were compared between the group of term, preterm ( $<37$ weeks), early preterm ( $<34$ weeks), and very early preterm ( $<32$ weeks) delivery. Predictive models were developed based on adjusted MoMs and logistic regression and diagnostic performances in predicting preterm birth were determined. $\boldsymbol{R e}$ sults: Of 20,780 pregnancies, 1,554 (7.5), 363 (1.7), and 158
}

$(0.8 \%)$ had preterm, early preterm, and very early preterm birth respectively. High levels of AFP and b-hCG but low levels of uE3 were significantly associated with higher rates of preterm, early preterm and very early preterm delivery. The predictive models had diagnostic performance in predicting preterm birth with the areas under the ROC curve of 0.688 , $0.534,0.599$, and 0.718 for AFP, b-hCG, uE3, and combined biomarkers respectively. Conclusion: The second trimester Down syndrome screening could also be used as a tool of risk identification of preterm birth in the same test, without extra-effort and extra-cost.

(C) 2019 S. Karger AG, Basel

\section{Introduction}

Preterm birth has historically been the leading cause of perinatal death worldwide, as well as in our country. A number of studies have attempted to find a way to facilitate the early prediction of preterm birth and provide effective measures to prevent preterm birth among higher-
Theera Tongsong, MD

Department of Obstetrics and Gynecology Faculty of Medicine, Chiang Mai University Chiang Mai 50200 (Thailand)

E-Mail theera.t@cmu.ac.th 
risk pregnancies, such as progesterone administration or cervical cerclage in some selected cases. Accordingly, the identification of the risk of preterm birth is essential.

Currently, the maternal serum biomarker screen for fetal Down syndrome has been extensively used, including in developing countries. Several studies have shown the association between individual biomarker levels and the risk of preterm birth [1-5], and some authors developed a predictive model derived from a combination of all biomarkers [6] to predict the risk of preterm birth. However, though many studies have shown the association between abnormal levels of second trimester biomarkers and preterm birth, most studies had a relatively small sample size, some results are conflicting, the study populations were heterogeneous, and finally, the results from most studies are hard to implement in clinical practice. Furthermore, ethnicity strongly impacts the level of biomarkers [7-10]. Thus, to take advantage of the serum screening test in clinical use, its interpretation should be based on its own reference range of the biomarkers. Additionally, the studies on the combination of all serum biomarkers as a predictor of preterm birth are very limited, and further studies on large-scale population are still needed. Therefore, we conducted this large populationbased study aimed to examine the correlation between the levels of serum biomarkers, as individual markers and their combination, and the risk of preterm birth and to develop a predictive model for clinical practice. We have been administrating fetal Down syndrome screening with maternal serum biomarkers for more than 7 years under the National Research University project. The second trimester triple screen, including alpha-fetoprotein (AFP), free beta-human chorionic gonadotropin (b-hCG), and unconjugated estriol ( $\mathrm{uE} 3)$, was a part of the project. In the project, all pregnancies were prospectively followed up until delivery for the pregnancy outcomes. The prospective database was developed, and data for serum markers and pregnancy outcomes were available. We aim to take advantage of second trimester serum marker screening to determine whether or not this screening can also predict the risk of preterm birth in late gestation.

\section{Materials and Methods}

This is a diagnostic test study conducted at the maternal-fetal medicine unit, Chiang Mai University, Thailand. It is a secondary analysis of the maternal-fetal medicine prospective database of the "Prenatal control of Down syndrome study" under the National Research University Project of Thailand, undertaken with ethical approval by the Institutional Review Boards.

Second Trimester Serum Screen as a

Predictor of Preterm Delivery
Database Development

In our previously mentioned prospective study on the prenatal control of Down syndrome, the database was developed. The main project was aimed at assessing the effectiveness of the maternal serum screen in our population including pregnant women attending our center and 32 community hospitals in northern Thailand. All women were prospectively followed up for a final diagnosis of Down syndrome and other pregnancy outcomes. All women had Thai ethnicity and resided in the northern part of Thailand. They participated with the project with written informed consent after receiving pretest counseling by our research team. The demographic data (including maternal age, weight, parity, ethnicity, smoking habits, and medical diseases), serum biomarker processing and transportation of the samples were audited by the research team and prospectively recorded. All blood samples were measured for biomarker concentrations at the same research center laboratory (fully automated immunoassay, DELFIA ${ }^{\circledR}$ Xpress system; Perkin Elmer, Waltham, MA, USA), with standard assay kits of AFP, beta-hCG and uE3. The screening tests were free of charge, as they were financially supported by the project. All participants were followed up for pregnancy outcomes such as abortion, fetal abnormalities, fetal growth, and maternal complications. All neonates were evaluated by the pediatricians on the research team. Cytogenetic studies were performed only in cases of high risk, as indicated by serum screening, or cases that were postnatally suspected of abnormalities after evaluation by the pediatricians. A diagnosis of aneuploidy was based on cytogenetic studies either by amniocentesis or postnatal work-up, while non-aneuploidy was based on cytogenetic studies or the conclusion by the pediatricians in cases of no cytogenetic study.

\section{Database Access}

During the study period from 2010 to 2016 , the database was accessed to retrieve all consecutive records with complete information for the demographic data and final pregnancy outcomes, including maternal age, maternal weight, smoking habits, medical disease, gestational age at the time serum was taken for biomarkers, gestational age at delivery, fetal birth weight and fetal chromosomal or structural abnormalities. The inclusion criteria were as follows: (1) having a singleton pregnancy, (2) undergoing the second trimester serum biomarker screen at 15-21 weeks of gestation, (3) having known pregnancy and fetal outcomes, and (4) having complete information for screening results and baseline characteristics. The exclusion criteria were as follows: (1) twin pregnancy, (2) fetal chromosomal or structural anomalies, (3) loss to follow-up or unknown pregnancy outcomes, (4) pregnancy ending abortion (20 weeks of gestation or less), and (5) serious maternal medical disease, such as cardiac disease with pulmonary hypertension, renal failure or uncontrolled severe hypertension, etc. Pregnancies meeting the inclusion criteria were categorized into 2 groups: the term delivery group (control group) and the preterm delivery group (study group). The study group was further subdivided into the early preterm group. Term delivery was defined as delivery at or after $37 \mathrm{com}$ plete weeks of gestation. Preterm delivery was defined as delivery before 37 complete weeks. Early preterm delivery was defined as delivery before completing 34 weeks of gestation. Very early preterm delivery was defined as delivery before completing 32 weeks of gestation. All records were reviewed for baseline 
characteristics, pregnancy outcomes and final diagnosis of fetal chromosomal and structural abnormalities. The primary outcomes of the study were rates of preterm birth and early preterm birth.

\section{Statistics}

The statistical analysis was performed using the statistical software package R and SPSS version 21.0 (IBM Corp. Released 2012; IBM SPSS Statistics for Windows, version 21.0. Armonk, NY, USA). The statistical procedures were similar to those used in identifying risk for fetal Down syndrome, as follows. The multiples of the median (MoMs) of each serum biomarker (AFP, b-hCG and uE3) were calculated by the following: (1) running a stepwise regression analysis of the $\log 10$ biomarker level as a dependent variable versus potential independent variables, such as gestational age at time of sampling, maternal weight, maternal age, smoking habits, and insulin-dependent diabetes mellitus; (2) calculating the expected $\log 10$ biomarker level for each woman based on the regression model created in the previous step; (3) converting the $\log 10$ value to be the expected level of the biomarker; and finally, (4) determining the MoM by dividing the measured biomarker level of each woman by her expected level. Preterm predictive models were created using logistic regression analysis, with biomarkers as dependent variables and term/preterm delivery as the independent variable. Based on the adjusted MoM values, the log-Gaussian distributions of each biomarker for term, preterm, and early preterm delivery were created. The performance of the predictive models was evaluated using ROC curves with sensitivities and false positive rates predicting preterm and early preterm births. The diagnostic performances of each biomarker and the combined markers were compared based on the area under curve. A likelihood ratio was then calculated by dividing the density in distribution of the preterm, early preterm and very early preterm group by that of the term group. Posterior (final) risk of preterm, early preterm and very early preterm birth was calculated by multiplying the likelihood ratio by the a priori risk.

\section{Results}

During the study period of our main Down syndrome screening project, 21,936 pregnancies were screened for second trimester serum biomarkers (AFP, b-hCG and $\mathrm{uE3}$ ), also known as the triple screen. After excluding 1,156 cases with various exclusion criteria, the remaining 20,780 were eligible for analysis, as presented in Figure 1. Of them, 19,226 pregnancies were assigned to the term delivery group, and 1,554 (7.5\%) were assigned to the preterm delivery group. The preterm delivery group included 363 cases $(1.7 \%)$ of early preterm births and 158 cases $(0.8 \%)$ of very early preterm births. The demographic data of both groups are presented in Table 1. Based on multiple regression analyses of the biomarkers (AFP, b-hCG and uE3 levels), the fitted models for expected levels of the 3 biomarkers were created, as follows:

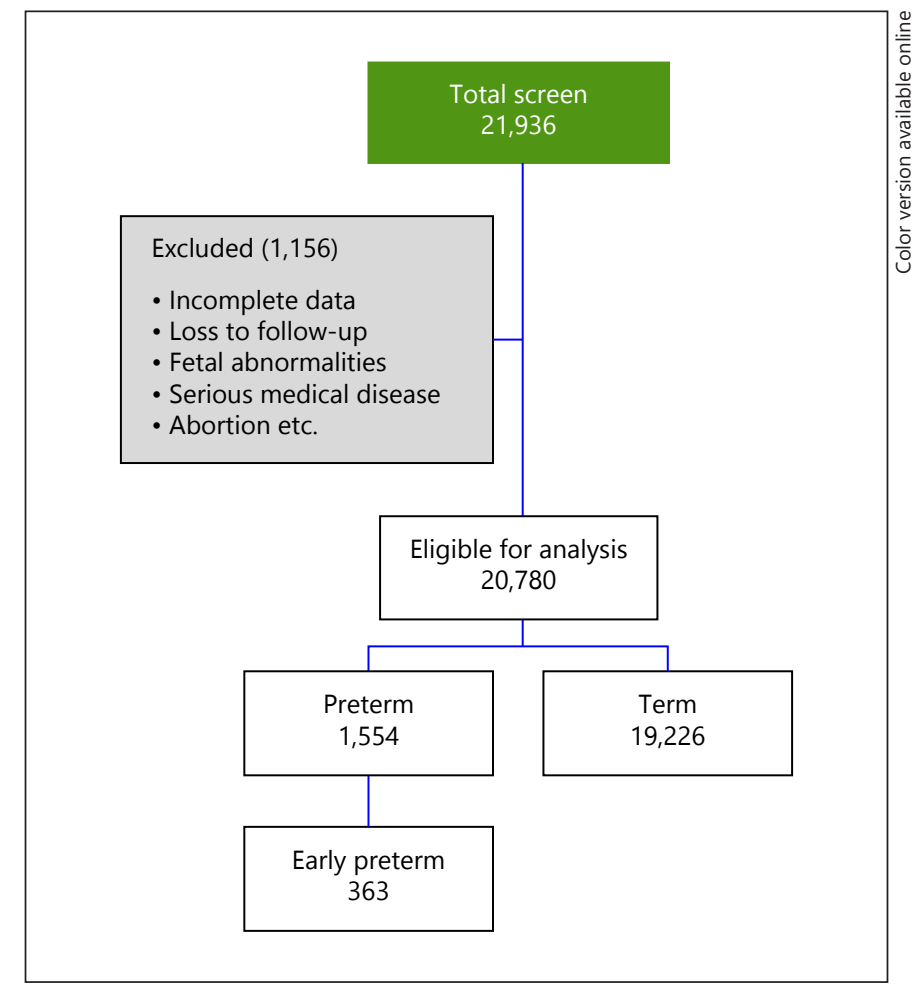

Fig. 1. Study flowchart.

Model of AFP levels: $\ln ($ AFP levels $)=0.631+0.070$ (GA: weeks) - 0.005 (weight: kg) +0.0005 (age, year) 0.014 (diabetes mellitus: DM); $(r=0.521 ; p$ value $<0.001)$

Model of b-hCG levels: ln (b-hCG levels) $=3.368-$ 0.112 (GA: weeks) - 0.006 (weight: $\mathrm{kg})-0.001$ (DM); $(r=$ 0.442 ; $p$ value $<0.001)$

Model of uE3 levels: $\ln (\mathrm{uE} 3$ levels $)=-1.124+0.117$ (GA: weeks) -0.002 (weight: $\mathrm{kg})-0.018(\mathrm{DM})+0.002$ (age, year); $(r=0.781 ; p$ value $<0.001)$

Note that maternal age and DM status are significantly associated with AFP and uE3 levels but not with b-hCG levels. The means and medians of the MoMs derived from the models from the 3 biomarkers for all women were compared between the term group and the preterm group, as well as the early preterm group, as presented in Table 2 and Figure 2. In terms of comparisons, the MoMs of AFP and b-hCG were significantly higher in the preterm/early preterm group than those in the controls, whereas the MoMs of uE3 were significantly lower in the preterm/early preterm group. Using logistic regression analysis, the models predicting preterm and early preterm delivery by each of the 3 biomarkers and their combination were constructed as follows: 


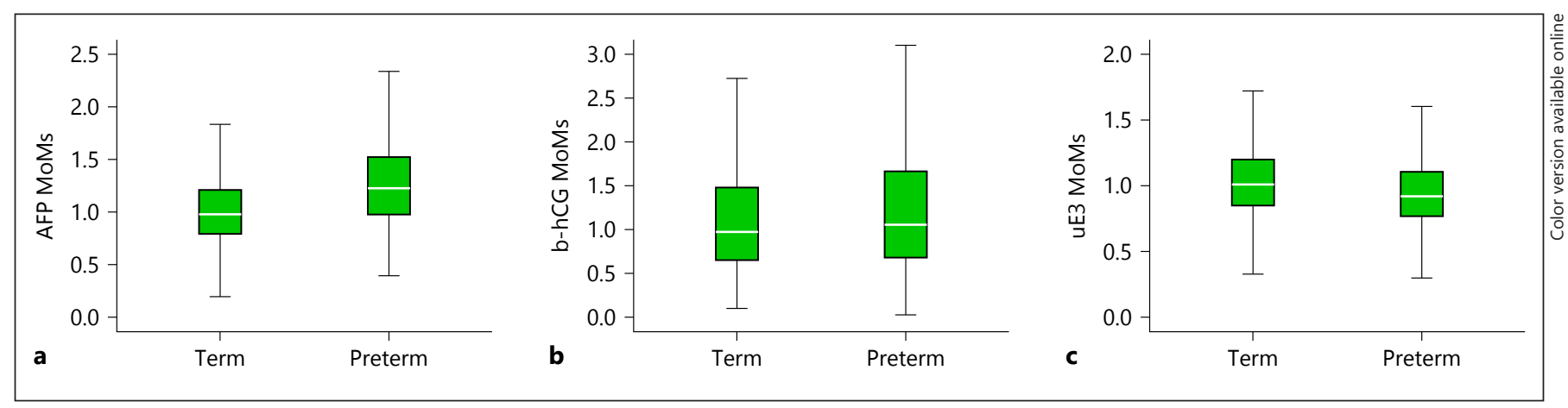

Fig. 2. Comparison of AFP MoMs (a), b-hCG MoMs (b) and uE3 (c) between term and preterm group. AFP, alpha-fetoprotein; MoMs, multiples of medians; b-hCG, beta-human chorionic gonadotropin; uE3, unconjugated estriol.

Table 1. Baseline characteristics of the pregnant women

\begin{tabular}{|c|c|c|c|c|c|c|c|}
\hline Characteristics & $\begin{array}{l}\text { Term } \\
\text { group }\end{array}$ & $\begin{array}{l}\text { Preterm } \\
\text { group }\end{array}$ & $p$ value* & $\begin{array}{l}\text { Early } \\
\text { preterm group }\end{array}$ & $p$ value* & $\begin{array}{l}\text { Very early } \\
\text { preterm group }\end{array}$ & $p$ value* \\
\hline Maternal age, years, mean \pm SD & $29.8 \pm 5.7$ & $30.7 \pm 5.8$ & $<0.001$ & $30.8 \pm 6.0$ & 0.002 & $31.6 \pm 5.8$ & 0.001 \\
\hline sampling, mean $\pm \mathrm{SD}$ & $15.9 \pm 1.0$ & $15.9 \pm 1.1$ & 0.276 & $15.8 \pm 1.0$ & 0.242 & $15.9 \pm 1.1$ & 0.870 \\
\hline Gestational week at & & & & & & & \\
\hline Parity, \% & & & 0.179 & & 0.104 & & 0.215 \\
\hline Nulliparous & 51.3 & 53.1 & & 55.6 & & 56.3 & \\
\hline Parous & 48.7 & 46.9 & & 44.4 & & 43.7 & \\
\hline
\end{tabular}

* Compared to the term group; using student $t$ test for continuous data, chi-square test for categorical data.

Table 2. Comparisons of mean and median MoMs of PAPP-A and b-hCG between term versus preterm, early preterm, and very early preterm group

\begin{tabular}{|c|c|c|c|c|c|c|c|}
\hline Group & Term & Preterm & $p$ value & Early preterm & $p$ value & $\begin{array}{l}\text { Very early } \\
\text { preterm }\end{array}$ & $p$ value \\
\hline \multicolumn{8}{|l|}{ Means $\pm \mathrm{SD}^{*}$} \\
\hline AFP MoMs & $1.040 \pm 0.437$ & $1.347 \pm 0.790$ & $<0.001$ & $1.468 \pm 0.839$ & $<0.001$ & $1.590 \pm 1.865$ & $<0.001$ \\
\hline b-hCG MoMs & $1.217 \pm 0.942$ & $1.360 \pm 1.056$ & $<0.001$ & $1.469 \pm 1.350$ & $<0.001$ & $1.486 \pm 1.293$ & 0.001 \\
\hline uE3 MoMs & $1.044 \pm 0.287$ & $0.951 \pm 0.274$ & $<0.001$ & $0.918 \pm 0.267$ & $<0.001$ & $0.903 \pm 0.251$ & $<0.001$ \\
\hline \multicolumn{8}{|l|}{ Median (IQR) \# } \\
\hline b-hCG MoMs & $0.972(0.83)$ & $1.222(0.86)$ & $<0.001$ & $1.091(1.06)$ & $<0.001$ & 1.095 (1.09) & 0.025 \\
\hline uE3 MoMs & $1.010(0.35)$ & $0.918(0.34)$ & $<0.001$ & $0.887(0.34)$ & $<0.001$ & $0.875(0.32)$ & $<0.001$ \\
\hline
\end{tabular}

* Student $t$ test.

\# Mann-Whitney U test (compared to “Term Group”).

AFP, alpha-fetoprotein; b-hCG, beta-human chorionic gonadotropin; uE3, unconjugated estriol; MoMs, multiples of the median.

Second Trimester Serum Screen as a Predictor of Preterm Delivery 
Table 3. The diagnostic performance of the 3 biomarkers in predicting preterm birth and early preterm birth

\begin{tabular}{lccc}
\hline Predictors & Preterm group & Early preterm group & Very early preterm group \\
\hline Detection rate at 5\% false-positive rate, \% & & & 17.6 \\
$\quad$ AFP levels & 17.8 & 9.6 & 18.4 \\
b-hCG levels & 7.3 & 12.9 & 10.8 \\
$\quad$ uE3 levels & 11.7 & 23.1 & 12.0 \\
$\quad$ Combined biomarkers & 21.6 & 28.4 & 20.9 \\
Detection rate at 10\% false-positive rate, \% & & 17.1 & 31.6 \\
$\quad$ AFP levels & 29.0 & 21.5 & 16.5 \\
b-hCG levels & 14.2 & 33.9 & 22.8 \\
$\quad$ uE3 levels & 39.6 & & 31.7 \\
Combined biomarkers & & \\
Area under curve (95\% CI) & $0.688(0.674-0.701)$ & $0.690(0.662-0.718)$ & $0.703(0.662-0.744)$ \\
AFP levels & $0.534(0.519-0.550)$ & $0.553(0.522-0.584)$ & $0.552(0.504-0.599)$ \\
b-hCG levels & $0.599(0.584-0.614)$ & $0.626(0.597-0.655)$ & $0.648(0.606-0.690)$ \\
uE3 levels & $0.718(0.705-0.732)$ & $0.717(690-0.744)$ & $0.715(0.675-0.755)$ \\
Combined biomarkers & & \\
\hline
\end{tabular}

AFP, alpha-fetoprotein; b-hCG, beta-human chorionic gonadotropin; uE3, unconjugated estriol.

Probability of Preterm (before 37 Weeks)

MoM of AFP levels: $\ln (\mathrm{OR})=-3.832-1.152$ (AFP

MoM)

MoM of b-hCG levels: $\ln (\mathrm{OR})=-2.670-0.121(\mathrm{~b}-\mathrm{hCG}$ MoM)

MoM of uE3 levels: $\ln (\mathrm{OR})=-1.195-1.327$ (uE3 MoM)

Combined biomarkers: $\ln (\mathrm{OR})=-2.373+1.274$ (AFP

MoM) + 0.026(b-hCG MoM) - 1.633 (uE3 MoM)

Probability of Early Preterm (before 34 Weeks)

MoM of AFP levels: $\ln (\mathrm{OR})=-4.875-0.735$ (AFP MoM)

MoM of b-hCG levels: $\ln (\mathrm{OR})=-4.237-0.157$ (b-hCG MoM)

MoM of uE3 levels: $\ln (\mathrm{OR})=-2.273-1.801$ (uE3 MoM)

Combined biomarkers: $\ln (\mathrm{OR})=-3.165+0.780$ (AFP

MoM) + 0.094(b-hCG MoM) -1.927 (uE3 MoM)

Probability of Very Early Preterm (before 32 Weeks)

MoM of AFP levels: $\ln (\mathrm{OR})=-5.393+0.453$ (AFP MoM)

MoM of b-hCG levels: $\ln (\mathrm{OR})=-5.075+0.153$ (b-hCG MoM)

MoM of uE3 levels: $\ln (\mathrm{OR})=-2.884-2.057$ ( $\mathrm{uE} 3$ MoM)

Combined biomarkers: $\ln (\mathrm{OR})=-3.536+0.475$ (AFP MoM) + 0.108(b-hCG MoM) -2.089 (uE3 MoM)
The diagnostic performances of the predictive models of the individual biomarkers as well as their combination in predicting preterm, early preterm and very early preterm birth are presented in Table 3 , and the ROC curves of the predictive models are presented in Figure 3. Note that as an individual biomarker, AFP has the highest predictive value, giving an area under curve of 0.688 and 0.690 for preterm and early preterm birth respectively. The higher MoMs of AFP, the higher the likelihood of preterm birth, as shown in Figure 4. The combination of the 3 biomarkers adds predictive value to yield a detection rate of more than 33\% for both preterm and early preterm birth with a $10 \%$ false positive rate (Table 3 ).

\section{Discussion}

Insights gained from this study are as follows: (1) each biomarker was significantly associated with an increase in preterm birth, early preterm and very early preterm birth rate; (2) of the 3 markers, AFP has the highest predictive value; (3) the combination of the 3 biomarkers gives a better diagnostic performance than an individual biomarker; and (4) the diagnostic performances of the biomarkers for predicting preterm, early preterm and very early preterm birth are comparable. The findings suggest that secondtrimester serum screening may be used as an adjunct in prediction of preterm delivery. Accordingly, the screen 


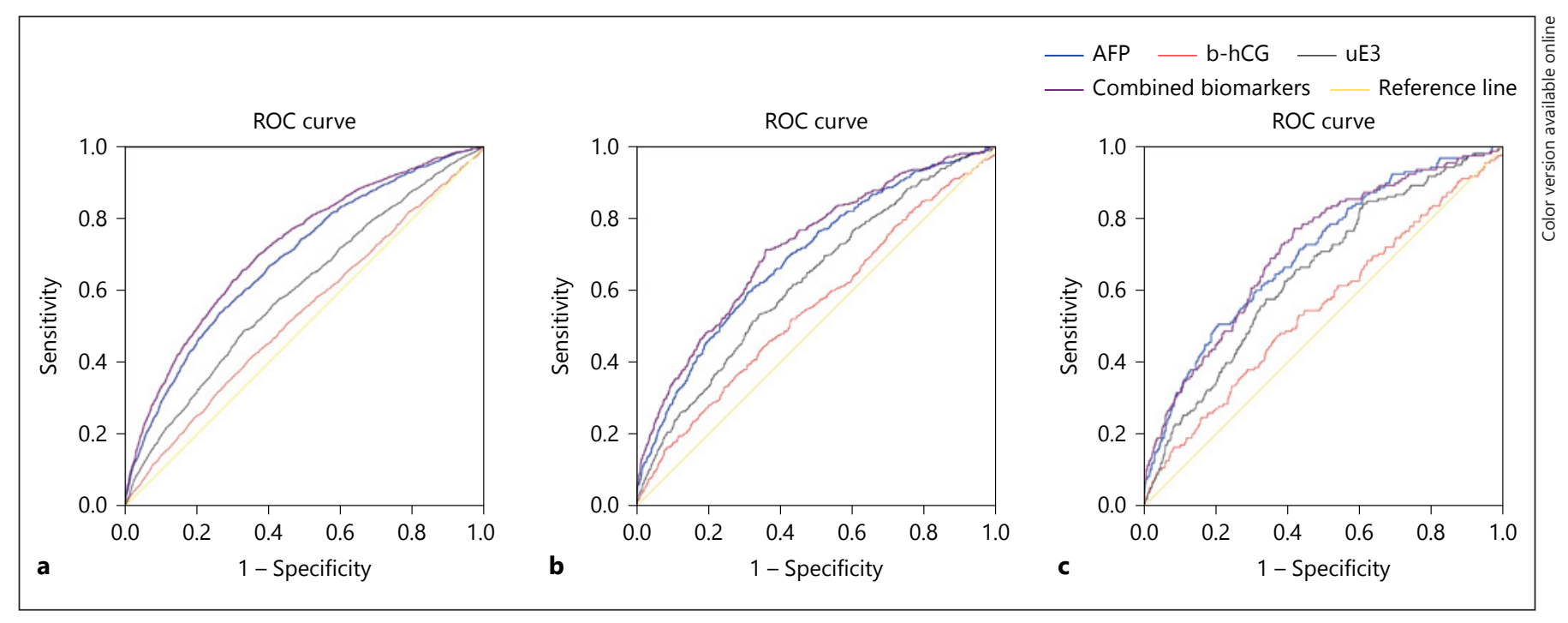

Fig. 3. ROC curves shows performance in predicting preterm (a), early preterm (b), and very early preterm (c) birth of the serum biomarkers. AFP, alpha-fetoprotein; b-hCG, beta-human chorionic gonadotropin; uE3, unconjugated estriol.

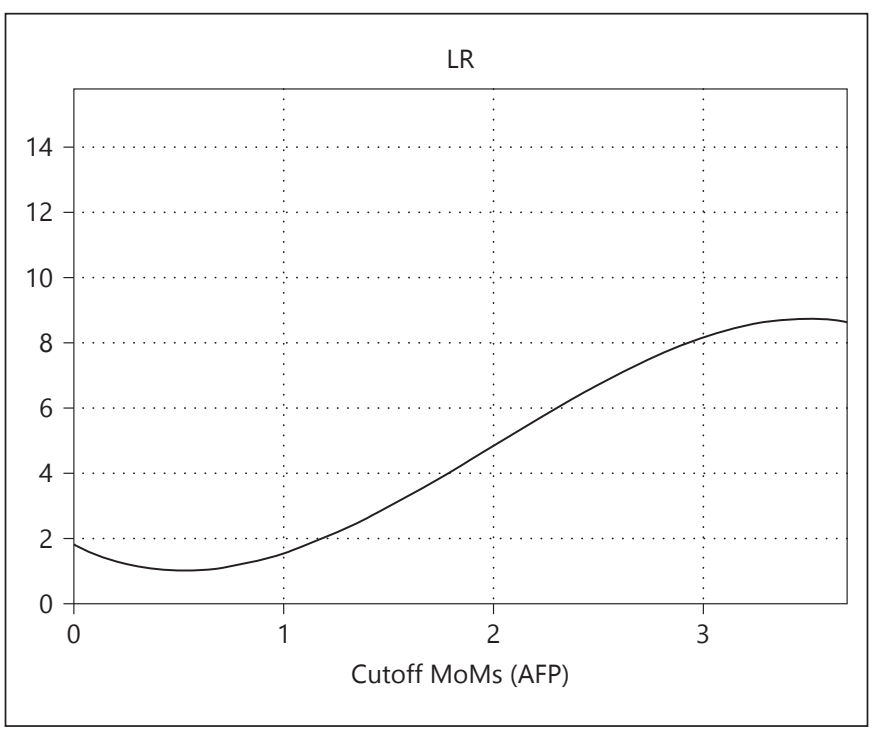

Fig. 4. Likelihood ratio for preterm birth based on MoMs of AFP. AFP, alpha-fetoprotein; MoMs, multiples of medians.

may be helpful in the case of selection for preterm prevention measures, such as special care at a high-risk clinic. The detection rate of preterm/early preterm birth by the biomarkers of routine second trimester screening does not seem to be very high, but this rate can probably modify the risk of preterm birth when used together with other background risk factors. Note that diagnostic performance improved with combination of the 3 biomarkers, when compared with that of the isolated one. Therefore, in clinical application, we suggest that the combination of the 3 biomarkers be used for preterm risk estimation. However, our study should be considered a preliminary one, and further studies in different populations should be evaluated for the reproducibility.

Our presentation of the relationship between biomarker levels and the risk of preterm birth is different from that of most previous studies $[1,2,4-6,11]$, in which the relative risk or ORs of preterm birth were presented when specific cut-offs of biomarker MoMs were used, such as an AFP of 2 MoMs or more or a uE3 of less than 0.5 MoMs. In contrast, we present the predictive models that could help us determine the individual risk for each woman, similar to the risk identification for Down syndrome. Furthermore, the general population risk of preterm birth could be further modified by the biomarker levels and our predictive model. For example if a woman is reported to have MoMs of AFP, hCG and $\mathrm{uE} 3$ of $2.8,1.5$ and 1.1 , respectively, then they are converted into a likelihood ratio of $3.0,1.2$, and 1.0 respectively. If the background risk (priori risk) of preterm labor in our population is $10 \%$, the woman has a risk (posterior risk) for preterm labor of $10 \times 3.0 \times 1.2 \times 1.1=$ $36 \%$. This estimate may be interpreted as high risk for preterm and early preterm birth and likely merits special care attention.

Our results show that serum markers for fetal Down syndrome screening in the second trimester could mod- 
ify the risk of preterm delivery. If the predictive models are incorporated into built-in software in the machine running serum biomarkers, the risk of preterm/early preterm birth can also be documented in the same reporting form as Down syndrome risk. Such reports could add valuable information for preterm prevention, though this association needs further confirmation by more extensive studies. When the predictive model for preterm delivery is incorporated, it can report as the likelihood ratio of individual biomarkers or posterior risk as reported for the risk of Down syndrome. The predictive models provided by this study are a simple tool for screening for the risk of preterm birth without any extra effort and extra cost (rather merely taking advantage of routine second trimester Down syndrome screening). This tool differs from other tools, such as transvaginal ultrasound to measure cervical length for preterm prediction, which is expensive, requires more expertise, and cannot be available for widespread use in developing countries.

The strengths of this study should be noted. First, the MoMs used to construct the predictive models were derived from our own population, and these MoMs are likely more appropriate than those derived from a Caucasian reference range with ethnic factor correction. Second, this study had high homogeneity in the study population, given that it included only individuals of Thai ethnicity. Third, the sample size was large enough to address the primary outcomes. Fourth, the predictive models derived from the study permitted us to calculate the individual risk of each woman or the individual likelihood ratio to multiply the background risk, rather than using one cutoff MoM value to indicate high or low risk as proposed in many previous studies. The models could be incorporated into the machine software generating automatic reports on the risk of preterm birth, simultaneously with the risk of Down syndrome. Fifth, because of the population-based design of the study, the models are more appropriate for generalization. Finally, the database was prospectively developed, and all pregnancy outcomes were followed up.

The weaknesses of this study should also be acknowledged. First, the main project of serum screening for Down syndrome did not include serum inhibin-A, which is one of the biomarkers in the quad test. Second, preterm births in this study included both spontaneous and indicated preterm births such as severe preeclampsia in early gestation. Therefore, though the models could predict the overall risk, they might be less useful for identifying risk of spontaneous preterm birth. Finally, it is possible that the models may not be properly applied to other ethnic groups that are much different from Thai population. Nevertheless, the results strongly suggest a relationship between biomarker concentrations and the risk of preterm delivery. We believe that our results may encourage other ethnic groups to develop models suitable for their own population.

\section{Acknowledgment}

This research was financially supported by the Faculty of Medicine, and Chiang Mai University Research Fund.

\section{Ethics Statement}

This study was funded by the Faculty of Medicine, and Chiang Mai University Research Fund. Informed consent was obtained from all individual participants included in this study. All of the authors declare that they have no conflict of interest. This study was conducted with the ethical approval of the institute review boards (Ethics Committee 4; Research ID 4981/Study Code: OBG2560-04981).

\section{Disclosure Statement}

The authors declare no conflicts of interest.

\section{Funding Source} Fund.

The Faculty of Medicine, and Chiang Mai University Research

\section{Synopsis}

The routine second trimester serum screening could also be used as a tool of risk identification of preterm birth.

References

1 Huerta-Enochian G, Katz V, Erfurth S: The association of abnormal alpha-fetoprotein and adverse pregnancy outcome: does increased fetal surveillance affect pregnancy outcome? Am J Obstet Gynecol 2001;184: 1549-1553.

2 Puntachai P, Wanapirak C, Sirichotiyakul S, Tongprasert F, Srisupundit K, Luewan S, et al: Associations between pregnancy outcomes and unexplained high and low maternal serum alpha-fetoprotein levels. Arch Gynecol Obstet 2015;292:81-85. 
3 Settiyanan T, Wanapirak C, Sirichotiyakul S, Tongprasert F, Srisupundit K, Luewan S, et al: Association between isolated abnormal levels of maternal serum unconjugated estriol in the second trimester and adverse pregnancy outcomes. J Matern Fetal Neonatal Med 2016;29: 2093-2097.

4 Duric K, Skrablin S, Lesin J, Kalafatic D, Kuvacic I, Suchanek E: Second trimester total human chorionic gonadotropin, alpha-fetoprotein and unconjugated estriol in predicting pregnancy complications other than fetal aneuploidy. Eur J Obstet Gynecol Reprod Biol 2003;110:12-15.

5 Walton DL, Norem CT, Schoen EJ, Ray GT, Colby CJ: Second-trimester serum chorionic gonadotropin concentrations and complications and outcome of pregnancy. N Engl J Med 1999;341:2033-38.
6 Cohen JL, Smilen KE, Bianco AT, Moshier EL, Ferrara LA, Stone JL: Predictive value of combined serum biomarkers for adverse pregnancy outcomes. Eur J Obstet Gynecol Reprod Biol 2014;181:89-94.

7 Bryant-Greenwood PK, O’Brien JE, Huang X, Yaron Y, Ayoub M, Johnson MP, et al: Maternal weight differences do not explain ethnic differences in biochemical screening. Fetal Diagn Ther 1998;13:46-48.

8 Manotaya S, Zitzler J, Li X, Wibowo N, Pham TM, Kang MS, et al: Effect of ethnicity on first trimester biomarkers for combined trisomy 21 screening: results from a multicenter study in six Asian countries. Prenat Diagn 2015;35: 735-740.
9 Spencer K, Heath V, El-Sheikhah A, Ong CY, Nicolaides KH: Ethnicity and the need for correction of biochemical and ultrasound markers of chromosomal anomalies in the first trimester: a study of Oriental, Asian and Afro-Caribbean populations. Prenat Diagn 2005;25:365-369.

10 Watt HC, Wald NJ, Smith D, Kennard A, Densem J: Effect of allowing for ethnic group in prenatal screening for down's syndrome. Prenat Diagn 1996;16:691-698.

11 Yaron Y, Cherry M, Kramer RL, O'Brien JE, Hallak M, Johnson MP, et al: Second-trimester maternal serum marker screening: maternal serum alpha-fetoprotein, beta-human chorionic gonadotropin, estriol, and their various combinations as predictors of pregnancy outcome. Am J Obstet Gynecol 1999; 181:968-974. 